\title{
因HAD
}

DOI: http://doi.org/10.22585/hospdomic.v2i4.54

\section{Prevalencia de Desnutrición en los pacientes de Hospitalización a Domicilio}

\section{Prevalence of Malnutrition in Home Hospitalization Patients}

Carmina Wanden-Berghe', Catalina Cases Alarcón', Nieves Santacruz Carmona', Arantxa García', Lourdes Granell', Teresa Gonzalez Valls', Marian Miralles'

1. Hospital General Universitario de Alicante. Instituto de Investigación Sanitaria y Biomédica de Alicante ISABIAL-FISABIO.

Correspondencia/Correspondence

Carmina Wanden-Berghe Lozano

Hospital General Universitario de Alicante,

Pintor Baeza sn, Alicante 03010

carminaw@telefonica.net

Recibido/Received

01.09 .2018

Aceptado/Accepted

22.09.2018
Conflicto de Intereses/Competing interest Las autoras, en su totalidad declaran que no hay ningún posible conflicto de interés.

Agradecimientos/Acknowledgments

Este trabajo fue presentado previamente a su publicación en forma de comunicación oral al 14 congreso de la Sociedad Española de Hospitalización a Domicilio, recibiendo la consideración del premio a la mejor comunicación.

Para su desarrollo recibió el apoyo de Nutricia Avanced Medical Nutrition.

Cómo CitAR ESTE tRAbajo | HOW TO CITE tHIS PAPER

Wanden-Berghe C, Cases Alarcón C, Santacruz Carmona N, García A, Granell L, Gonzalez Valls T, et al. Prevalencia de Desnutrición en los pacientes de Hospitalización a Domicilio. Hosp Domic. 2018;2(4):137-43. 


\section{RESUMEN}

Objetivo: Estimar la prevalencia de desnutrición en los pacientes ingresados en la UHD del Hospital General Universitario de Alicante.

Material y Métodos: Estudio longitudinal prospectivo de una muestra de 386 pacientes ingresados en UHD incluidos aleatoriamente. Los pacientes fueron evaluados con la Herramienta de Evaluación Nutricional HEN [https://www. evalnut.com/]. Durante el año 2016-2017.

Resultados: Los 386 pacientes tenían una media de edad de 72,87 años y $51,7 \%$ eran varones. El $42,1 \%$ de los pacientes tenían un nivel de enfermedad "agudo leve / crónico estable" (Fig1). Eran portadores de gastrostomía 24(9,2\%). No cubría el $75 \%$ de los requerimientos energéticos diarios el 42,1\%. Habían perdido peso antes de su ingreso en $\mathrm{HaD}$ el $69,7 \%$ de los pacientes [media $10,1 \pm 6,1 \%$ de su peso habitual] independiente del sexo $(p=0,343)$ y la edad $(p=0,712)$ presentando mayor pérdida los que lo habían hecho en más de 1 mes $(p<0,001)$, también según se incrementa la gravedad de la enfermedad $(p<0,001)$ y sin relación con la disminución de la ingesta declarada $(p=0,395)$. Tenían dificultades para masticar $21,5 \%$, presentaban disfagia 18,4\% (Fig2) de los que 91,3\% era a líquidos. El 91,6\% de los pacientes tenía algún tipo de desnutrición, siendo leve el 54,4\% y destacando la desnutrición mixta en el 71,3\% de los casos (Fig3). No se encontró relación entre la pérdida de peso y el tipo de desnutrición $(p=0,054)$ y sí entre el nivel de gravedad de la enfermedad y con el tipo de desnutrición y grado de desnutrición $(p=0,005 ; p=0,034)$.

Conclusiones: Los pacientes ingresados en HaD presentan una altísima prevalencia de desnutrición, mayoritariamente de tipo mixta, siendo ésta más severa a medida que se incrementa la gravedad de la enfermedad.

La relación entre el grado y tipo de desnutrición con la gravedad de la enfermedad hace pensar que se ha ido instaurando durante un largo periodo de tiempo.

Palabras clave: Desnutrición; Hospitalización a Domicilio; Prevalencia

\section{ABSTRACT}

Objective: To estimate the prevalence of malnutrition in patients admitted to the UHD of the General University Hospital of Alicante.

Material and methods: Prospective longitudinal study of a sample of 386 patients admitted to UHD randomly selected. Patients were evaluated with the HEN Nutritional Assessment Tool [https://www.evalnut.com/]. During the years 2016-2017.

Results: The 386 selected patients had an average age of 72.87 years. 51.7 percent of them were male and 42.1 percent had a level of illness "acute mild / chronic stable" (Fig. 1). 24 of the patients (9.2 percent) were gastrostomy carriers. 42.1 percent of patients did not cover 75 percent of daily energy requirements. 69.7 percent of patients lost weight before admission in the $\mathrm{HaD}$ [mean $10.1 \pm 6.1$ percent of their usual weight], independent of $\operatorname{sex}(p=0.343)$ and age $(p=0.712)$, presenting higher lost weight those who had done for more than a month ( $p$ $<0.001)$, as well as those in whom the severity of the disease had increased $(p<0.001)$ and not related to the decrease in reported intake $(p$ $=0.395$ ). 21.5 percent of patients had difficulty chewing and 18.4 percent of them had dysphagia (Fig2) of which 91.3 percent was from liquids. 91.6 percent of patients had some type of malnutrition, of which 54.4 percent were mild, highlighting that 71.3 percent of cases were mixed malnutrition (Fig 3). No relationship was found between the weight loss and the type of malnutrition ( $p=0.054$ ). However a relationship between the severity level of the disease and the type and degree of malnutrition ( $p=0.005, p=$ 0.034) was found.

Conclusions: Patients admitted to $\mathrm{HaD}$ have a very high prevalence of malnutrition, mostly of mixed type, which is more severe as the severity of the disease increases. The relationship between the degree and type of malnutrition with the severity of the disease suggests that it has been established for a long period of time.

Keywords: Malnutrition; Home Care Services, Hospital-Based; Prevalence. 


\section{INTRODUCCIÓN}

La desnutrición relacionada con la enfermedad se define como una alteración de la composición corporal producida por un déficit de nutrientes (1), como consecuencia de una enfermedad aguda o crónica, que disminuye las funciones vitales y repercute de forma negativa en la evolución clínica.

Los pacientes de las Unidades de Hospitalización a Domicilio (UHD) necesitan una asistencia sanitaria caracterizada por cuidados de intensidad y complejidad equiparable a los dispensados en el hospital $(2,3)$. Proceden de todos los niveles asistenciales (hospital de agudos, urgencias hospitalarias, residencias geriátricas, atención primaria, hospitales de larga y media estancia). La prevalencia de la desnutrición es diferente en los distintos niveles asistenciales por lo tanto en las UHD estaríamos recibiendo pacientes procedentes de todos estos colectivos y se podría ponderar para conocer la prevalencia en dichas Unidades. Pero no sería real, ya que los pacientes que llegan a UHD están seleccionados en función de su complejidad, pluripatología, fragilidad, grado de descompensación, e incluso de su terminalidad, además de una serie de variables que podrían afectar en su estado nutricional. También el hecho de que la edad media de estos pacientes sea generalmente muy avanzada 77,45 \pm 11 años (4), Es un hecho la relación del envejecimiento progresivo de la población con la desnutrición y con el riego de desnutrición, y de su relación con la discapacidad, siendo reconocida como la causa más frecuente de discapacidad en la población geriátrica que vive en su propio hogar o en instituciones (5-7)

La desnutrición en Hospitalización a Domicilio HaD continúa siendo un problema de dimensiones no bien conocidas, es por ello que este trabajo se propone estimar la prevalencia de desnutrición en una muestra suficientemente representativa de los pacientes ingresados en la UHD del Hospital General Universitario de Alicante para que permita extrapolar los resultados a otras unidades que sean similares en el tipo de pacientes.

\section{MÉTODOS}

Estudio longitudinal prospectivo de pacientes ingresados en UHD del hospital general universitario de Alicante. El muestreo fue aleatorio simple durante el año 2016-2017 y el tamaño de la muestra se calculó mediante la estimación de parámetros poblacionales en una población infinita, considerando la prevalencia publicada en el estudio PREDYCES (8) «Uno de cada cuatro pacientes hospitalizados está en riesgo de desnutrición o desnutrido (23,7 \%)» (prevalencia=23,7\%; precisión del intervalo=0,05; nivel de confianza $=0,95$ ) con una proporción esperada de pérdidas del $15 \%$, siendo de 328 pacientes.

Criterios de inclusión: Pacientes que ingresen en la $\mathrm{HaD}$ desde cualquier procedencia (hospital de agudos, hospital de larga -media estancia, atención primaria, urgencias o residencias geriátricas) y pertenecientes a cualquier grupo patológico que hayan firmado (ellos o su representante legal) el consentimiento informado para participar en el estudio.

Criterios de exclusión: pacientes que reingresen en $\mathrm{HaD}$ y que ya hayan sido previamente incluidos en el estudio.

Los pacientes fueron evaluados con la Herramienta de Evaluación Nutricional HEN https://www. evalnut.com/ para conocer su estado nutricional. La HEN recoge datos dietéticos, antropométricos estáticos y evolutivos, además de analíticos. Calcula el estado nutricional según algoritmos definidos por los autores en base a una exhaustiva revisión de las publicaciones científicas y de acuerdo con los consensos y parámetros definidos por las Sociedades Científicas ESPEN, SENPE y ASPEN. Define el tipo de malnutrición y su grado. 
Para la descripción de la población estudiada se utilizaron técnicas descriptivas; cálculo de frecuencias y, en el caso de las cuantitativas, medidas de tendencia central y desviación estándar (SD) o el intervalo intercuartílico (IIQ), cuando así lo aconsejó la distribución. Para el análisis entre variables, se utilizó los estadísticos Chi-cuadrado, t de Student y ANOVA.

\section{RESULTADOS}

Se incluyeron 386 pacientes que tenían una edad media de 72,87años de los que el 51,7\% eran varones. El 90\% de los pacientes pertenecían al grupo patológico médico y el 10\% al quirúrgico. $42,1 \%$ de los pacientes tenían un nivel de enfermedad "agudo leve / crónico estable" (Figura 1). Eran portadores de gastrostomía el 9,2\%. Estaban comiendo tres cuartas partes menos respecto a su alimentación habitual el $16,5 \%$ de los pacientes y el $42,1 \%$ no cubría el $75 \%$ de sus requerimientos energéticos diarios (Figura 2). Habían perdido peso antes de su ingreso en $\mathrm{HaD}$ el 69,7\% de los pacientes [media 10,1 $\pm 6,1 \%$ de su peso habitual] independiente del sexo $(p=0,343)$ y la edad $(p=0,712)$ presentando mayor pérdida los que lo habían hecho en más de 1 mes $(p<0,001)$, también fue mayor la pérdida de peso según se incrementa la gravedad de la enfermedad $(p<0,001)$ y sin relación con la disminución de la ingesta declarada $(p=0,395)$. Tenían dificultades para masticar $21,5 \%$, presentaban disfagia $18,4 \%$ de los que $91,3 \%$ era a líquidos y el $50 \%$ moderada (Figura 3). El 91,6\% de los pacientes tenía algún tipo de desnutrición [IC 95\% 88,9 - 94,4\%], siendo leve el $54,4 \%$ y destacando la desnutrición mixta en el $71,3 \%$ de los casos (Figura 4). No se encontró relación entre la pérdida de peso y el tipo de desnutrición $(p=0,054)$ y sí entre el nivel de gravedad de la enfermedad y con el tipo de desnutrición y grado de desnutrición ( $p=0,005 ; p=0,034$ ). Tabla 1 .

Figura 1. Nivel de gravedad de la enfermedad

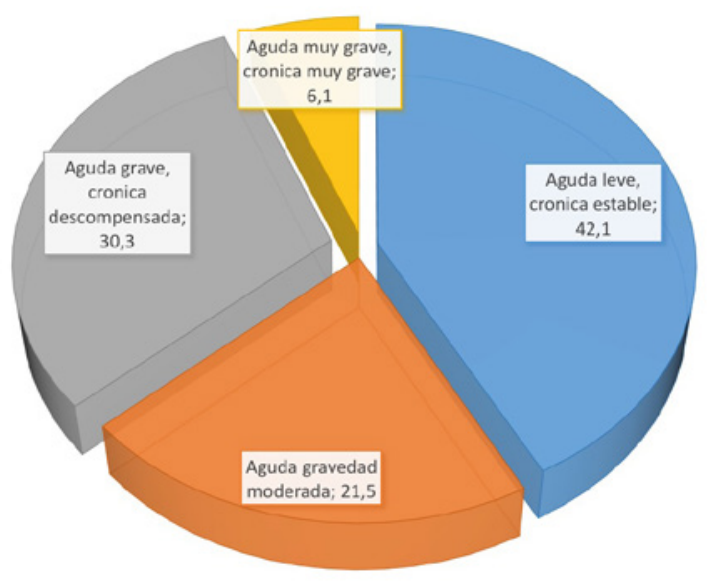


Figura 2. Historia dietética

\section{Respecto a su alimentación habitual esta tomando(\%)}

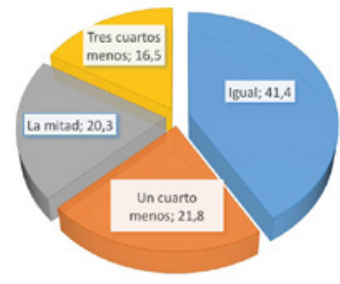

Cubre al menos el $75 \%$ de los requerimientos diarios (\%)

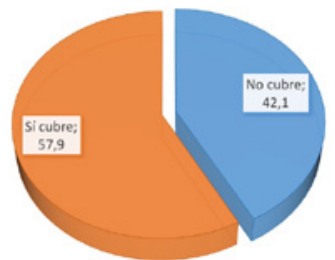

Figura 3. Disfagia, tipo y gravedad (\%)

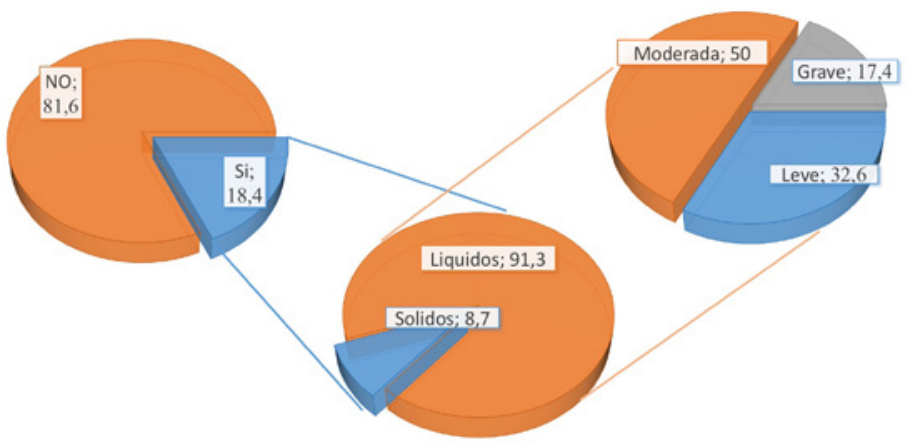

Figura 4. Desnutrición y tipo y severidad (\%)

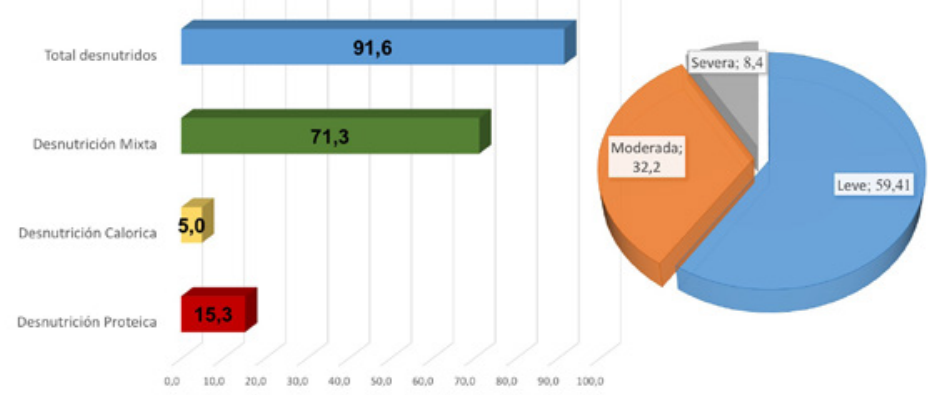


Tabla 1. Relación observada entre el \% de perdida de peso respecto al peso habitual y con la gravedad de la enfermedad

\begin{tabular}{l|l|l|l}
$\%$ & Media \pm sd & Variables de contraste & p-valor
\end{tabular}

\begin{tabular}{|c|c|c|c|c|}
\hline \multirow{6}{*}{$\begin{array}{l}\% \text { Perdida del peso } \\
\text { habitual al ingreso }\end{array}$} & \multirow{6}{*}{69,7} & \multirow{6}{*}{$-10,1 \pm 6,1 \%$} & Sexo & 0,343 \\
\hline & & & Edad & 0,712 \\
\hline & & & En más de 1 mes & $<0,001^{*}$ \\
\hline & & & Gravedad de la enfermedad & $<0,001^{*}$ \\
\hline & & & $\begin{array}{l}\text { Disminución de la ingesta } \\
\text { declarada }\end{array}$ & 0,395 \\
\hline & & & Tipo de desnutrición & $0,054^{*}$ \\
\hline \multirow{2}{*}{\multicolumn{2}{|c|}{ Gravedad de la enfermedad }} & & Grado de desnutrición & $0,034 *$ \\
\hline & & & Tipo de desnutrición & $0,005^{*}$ \\
\hline
\end{tabular}

\section{DISCUSIÓN}

Destaca en este estudio la altísima prevalencia de desnutrición en los pacientes que ingresan en UHD, se podría decir que prácticamente todos tienen algún grado de desnutrición. Este hecho no es extraño si tenemos en cuenta que a este Servicio se derivan pacientes con perfil más complejo; pluripatológicos, evoluciones complicadas y tórpidas y pacientes paliativos en estadíos muy avanzados de la enfermedad. No podemos decir que este hecho sea un indicador de que el estado nutricional de estos pacientes no haya sido uno de los principales objetivos en sus tratamientos, se necesitaría otro tipo de diseño de estudio para responder a esta pregunta y no ha sido contemplada como un objetivo en este trabajo. Tampoco podemos decir que en los pacientes que tenían prescrita suplementación o nutrición artificial esta no estuviera ofreciendo los resultados esperados, por el mismo motivo anteriormente expuesto.

Es conocido que los pacientes con desnutrición presentan más complicaciones y tienen evoluciones complejas, si ingresan en el hospital tienen estancias hospitalarias más prolongada, reciben más tratamientos y polifarmacia que como hemos visto conforman el subgrupo de pacientes que son subsidiarios de ingreso en Hospitalización a Domicilio.

Un trabajo realizado en 2006 (4), en tres hospitales comarcales de la Comunidad Valenciana con un total de 40 pacientes incluidos, valoró el estado nutricional mediante el cuestionario de Valoración Global Subjetiva encontraron un 50\%.

Se podría considerar que en el presente estudio con una mayor muestra, ofrece una imagen de la realidad nutricional de los pacientes de UHD más realista o bien se podría especular con la idea de que el perfil de los pacientes que ingresan en la UHD, en los 12 años que separan ambos trabajos, ha evolucionado hacia un paciente cada vez más complejo y deteriorado a pesar de haber sido clasificada su patología en el $42,1 \%$ como "agudo leve / crónico estable", si bien hay que tener en cuenta que esta clasificación obedece a determinar el incremento energético estimado para calcular sus requerimientos calóricos y no a la complejidad de sus patologías. 
Por tanto, se puede concluir: Los pacientes ingresados en $\mathrm{HaD}$ presentan una altísima prevalencia de desnutrición, mayoritariamente de tipo mixta, siendo ésta más severa a medida que se incrementa la gravedad de la enfermedad.

La relación entre el grado y tipo de desnutrición con la gravedad de la enfermedad hace pensar que se ha ido instaurando durante un largo periodo de tiempo.

\section{BIBLIOGRAFÍA}

1. Stratton RJ, Green CJ, Elia M. Disease-related malnutrition: an evidence-based approach to treatment. Wallingford, United Kingdom: CABI Publishing; 2003.

2. Ruipérez Cantera I. Hospitalización a domicilio. Rev Clin Esp. 2000;200(6):299-300. PMID: 10953580

3. Massa Domínguez B. La hospitalización a domicilio en el siglo XXI. Hosp Domic. 2017;1(1):7-9. DOI: 10.22585/hospdomic.v1i1.8

4. Wanden-Berghe C, Sanz-Valero J, Jimenez M, Hernandez M. Desnutrición en los enfermos de Hospitalización a Domicilio. Nutr Hosp. 2006;21(1):22-5. PMID: 16562808

5. Cralsson M, Gustafson Y, Erickson S, Haglin L. Body composition in Swedish old people aged 65-99 years, living in residential care facilities. Arch Gerontol Geriatr. 2009;49(1):98-107. DOI: 10.1016/j.archger.2008.05.012; PMID: 18703240

6. García de Lorenzo y Mateos A, Álvarez J, De Man F. Envejecimiento y desnutrición; un reto para la sostenibilidad del SNS: conclusiones del IX Foro de Debate Abbott-SENPE. Nutr Hosp 2012;27(4):1060-4. DOI: 10.3305/nh.2012.27.4.5979; PMID: 23165542

7. García-Lorda P, Foz M, Salas-Salvadó J. Estado nutricional de la población anciana de Cataluña. Med Clin (Barc) 2002;118(18):707-15. PMID: 12042138

8. Álvarez-Hernández J, Planas Vila M, León-Sanz M, García de Lorenzo A, Celaya-Pérez S, García-Lorda P, et al. Prevalencia y costos de la desnutrición en pacientes hospitalizados; el estudio PREDYCES. Nutr Hosp. 2012;27(4):1049-59. DOI: 10.3305/nh.2012.27.4.5986; PMID: 23165541 